PROCEEDINGS OF THE

AMERICAN MATHEMATICAL SOCIETY

Volume 139, Number 1, January 2011, Pages 11-19

S 0002-9939(2010)10584-1

Article electronically published on August 18, 2010

\title{
HIGHER DIOPHANTINE APPROXIMATION EXPONENTS AND CONTINUED FRACTION SYMMETRIES FOR FUNCTION FIELDS
}

\author{
DINESH S. THAKUR
}

(Communicated by Matthew A. Papanikolas)

\begin{abstract}
We construct many families of nonquadratic algebraic Laurent series with continued fractions having a bounded partial quotients sequence (the diophantine approximation exponent for approximation by rationals is thus 2 , agreeing with the Roth value) and with the diophantine approximation exponent for approximation by quadratics being arbitrarily large. In contrast, the Schmidt value (analog of the Roth value for approximations by quadratics, in the number field case) is 3 . We calculate diophantine approximation exponents for approximations by rationals for function field analogs of $\pi, e$ and Hurwitz numbers (which are transcendental) and also give an interesting lower bound (which may be the actual value) for the exponent for approximation by quadratics for the latter two. We do this exploiting the situation when 'folding' or 'negative reversal' patterns of the relevant continued fractions become 'repeating' or 'half-repeating' in even or odd characteristic respectively.
\end{abstract}

\section{BACKGROUND}

We recall [S80, Chapter 8] some basic definitions, facts and conjectures about the diophantine approximation of real numbers by rationals or (real) algebraic numbers. (See also [B04, BG06] and W] for a nice survey of recent developments.)

Definition 1 (Absolute and field height). For $\beta$ a nonzero algebraic number, define $H(\beta)$ to be the maximum of the absolute values of the coefficients of a nontrivial irreducible polynomial with co-prime integral coefficients that it satisfies. For $\beta$ lying in a number field $L$, define $H_{L}(\beta)$ similarly by replacing the irreducible polynomial by $c \prod\left(x-\beta^{(i)}\right)$, where the $\beta^{(i)}$ are its field conjugates and the multiple $c$ makes the coefficients co-prime integers.

Let $L$ be a number field inside $\mathbb{R}$ and let $d$ be a positive integer.

Definition 2 (Higher diophantine approximation exponents). For $\alpha$ an irrational real number not algebraic of degree $\leq d$, define $E_{d}(\alpha)\left(E_{\leq d}(\alpha)\right.$ respectively) as $\lim \sup (-\log |\alpha-\beta| / \log H(\beta))$, where $\beta$ varies through all algebraic real numbers of degree $d$ ( $\leq d$ respectively).

Received by the editors January 2, 2010.

2010 Mathematics Subject Classification. Primary 11J68, 11J70, 11J93.

This research was supported in part by NSA grant H98230-08-1-0049.

(C)2010 Dinesh S. Thakur 
For $\alpha \in \mathbb{R}-L$, define $E_{L}(\alpha)$ by the same formula but with $H$ replaced by $H_{L}$ and with $\beta$ varying through elements of $L$. Note that $E_{1}(\alpha)=E_{\mathbb{Q}}(\alpha)$ is the usual exponent $E(\alpha):=\lim \sup (-\log |\alpha-P / Q| / \log |Q|)$.

Then for irrational $\alpha$, we have $E(\alpha) \geq 2$ by Dirichlet's theorem, whereas for irrational algebraic $\alpha$ of degree $d$, we have $E(\alpha) \leq d$ by Liouville's theorem and $E(\alpha)=2$ by Roth's celebrated theorem improving Liouville, Thue, Siegel, and Dyson bounds.

For real $\alpha$ not in $L$, we have $E_{L}(\alpha) \geq 2$ and for real algebraic $\alpha$ not in $L$, we have $E_{L}(\alpha)=2$ by Leveque's generalization of Roth's theorem.

For real $\alpha$ not algebraic of degree $\leq d$, Wirsing (generalizing Dirichlet's result) conjectured (see also [R03]) $E_{\leq d}(\alpha) \geq d+1$ and proved a slightly better lower bound than $(d+3) / 2$, whereas Davenport and Schmidt proved his conjecture for $d=2$. On the other hand, for $\alpha$ of degree $>d$, we have Liouville's bound $E_{\leq d}(\alpha) \leq \operatorname{deg} \alpha$. Schmidt (generalizing Roth's result) proved that for real algebraic $\alpha$ of degree greater than $d, E_{\leq d}(\alpha) \leq d+1$.

From now on unless stated otherwise, we only focus on the function field analogs (see e.g. [T04 for a general background and [T04, Cha. 9], [T09] for the diophantine approximation, continued fractions background and references), where the roles of $\mathbb{Z}, \mathbb{Q}, \mathbb{R}$ are given by $A=\mathbb{F}_{q}[t], K=\mathbb{F}_{q}(t), K_{\infty}=\mathbb{F}_{q}((1 / t))$ respectively, where $\mathbb{F}_{q}$ is a finite field of characteristic $p$ containing $q$ elements. With the usual absolute value coming from the degree in $t$ of polynomials or rational functions, we have exactly similar definitions of heights and exponents. Now by rationals, reals, algebraic, we mean elements of $K, K_{\infty}$ and algebraic over $K$ respectively.

Then analogs of Dirichlet's and Liouville's theorems hold, but the naive analog of Roth's theorem fails, as shown by Mahler [M49]. For other results, see dM70, S00, T04 and references there, for example, results KTV00 in the Wirsing direction.

\section{Continued Fractions}

Continued fractions are natural tools of the theory of diophantine approximation. See dM70, BS76, S00, T04 for the basics in the function field case.

Let us review some standard notation. We write $\alpha=a_{0}+1 /\left(a_{1}+1 /\left(a_{2}+\cdots\right)\right)$ in the short form $\left[a_{0}, a_{1}, \cdots\right]$. We write $\alpha_{n}=\left[a_{n}, a_{n+1}, \cdots\right]$, so that $\alpha=\alpha_{0}$. Let us define $p_{n}$ and $q_{n}$ as usual in terms of the partial quotients $a_{i}$, so that $p_{n} / q_{n}$ is the $n$-th convergent $\left[a_{0}, \cdots, a_{n}\right]$ to $\alpha$. Hence $\operatorname{deg} q_{n}=\sum_{i=1}^{n} \operatorname{deg} a_{i}$.

Following the basic analogies mentioned above, we use the absolute value coming from the degree in $t$, and to generate the continued fraction in the function field case, we use the 'polynomial part' in place of the 'integral part' of the 'real' number $\alpha \in K_{\infty}$. In the function field case, for $i>0, a_{i}$ can be any nonconstant polynomial, and so the degree of $q_{i}$ increases with $i$, but $a_{i}$ or $q_{i}$ need not be monic. As usual, we have

$$
p_{n} q_{n-1}-q_{n} p_{n-1}=(-1)^{n-1}, \quad \alpha=\frac{\alpha_{n+1} p_{n}+p_{n-1}}{\alpha_{n+1} q_{n}+q_{n-1}}
$$

implying the usual basic approximation formula

$$
\alpha-p_{n} / q_{n}=(-1)^{n} /\left(\left(\alpha_{n+1}+q_{n-1} / q_{n}\right) q_{n}^{2}\right),
$$

which because of the non-Archimedean nature of the absolute value now implies

$$
\left|\alpha-p_{n} / q_{n}\right|=1 /\left(\left|a_{n+1}\right|\left|q_{n}\right|^{2}\right) \text {. }
$$


If we know the continued fraction for $\alpha$, the equation allows us to calculate the exponent, using $\operatorname{deg} q_{n}=\sum_{1}^{n} \operatorname{deg} a_{i}$, as

$$
E(\alpha)=2+\lim \sup \frac{\operatorname{deg} a_{n+1}}{\sum_{i=1}^{n} \operatorname{deg} a_{i}} .
$$

\section{Exponents of ANALOGS OF $e$ AND HuRWitz NUMBERS}

First we calculate (see also [T96]) exponents of analogs of Hurwitz numbers $\left(a e^{2 / n}+b\right) /\left(c e^{2 / n}+d\right)$, using Carlitz-Drinfeld exponential $e(z)$ [T04, T96, T97]. It is enough to calculate (by invariance of exponent under integral Mobius transformation of nonzero determinant) the exponent of $e(1 / f)$. (We leave the case of the analog of $e^{2 / n}$ discussed in [T96, 4.7] and [T97] to the interested reader.) These analogs of Hurwitz numbers are transcendental [T04, Chapter 10].

Theorem 1. Let $e(z)$ be the Carlitz-Drinfeld exponential function for the Carlitz $A$-module. Then for $f \in A-0$, we have the $E(e(1 / f))=q$.

Proof. Define $[n]=t^{q^{n}}-t, D_{0}=1$ and $D_{n+1}=[n] D_{n}^{q}$. Then by T92, we have the continued fraction expansion of $e(1 / f)-1 / f$ as $\lim \left[0, X_{n}\right]$ where $X_{1}=\left[f^{q}[1]\right]$ and $X_{n+1}=\left[X_{n},-f^{q^{n}(q-2)} D_{n+1} / D_{n}^{2},-X_{n}^{-}\right]$. Hence if we write $d_{n}$ for the degree of the denominator of $\left[0, X_{n}\right]$ and the degree of $f$ as $F$, then we see that $d_{1}=q(F+1)$ and $d_{n+1}=2 d_{n}+q^{n}(q-2) F+q^{n} n(q-2)+q^{n+1}$, so that $d_{n}=(n+F) q^{n}$ and the exponent formula above gives the exponent as limit of $2+\left(q^{n}(q-2) F+(n+\right.$ 1) $\left.q^{n+1}-2 n q^{n}\right) /\left((n+F) q^{n}\right)$.

\section{Folding LEMMA}

The continued fraction expansion for the exponential above and many calculations below are based on the following simple lemma, called the folding lemma, due to Mendes France [MF73], which has been rediscovered many times.

Lemma 1. Let $\left[a_{0}, a_{1}, \cdots, a_{n}\right]=p_{n} / q_{n}$, with the usual notation of continued fractions. Then $\left[a_{0}, \cdots, a_{n}, y,-a_{n}, \cdots,-a_{1}\right]=p_{n} / q_{n}+(-1)^{n} / y q_{n}^{2}$.

This pattern is a signed block reversal/folding pattern with the new term $y$. We will use the short form $-X^{-}$for the tuple $\left(-a_{n}, \cdots,-a_{1}\right)$ where $X$ is the tuple $X=\left(a_{1}, \cdots, a_{n}\right)$.

Consider the recurring folding type

$$
\begin{array}{r}
\alpha=\left[0, X_{1}, y_{1},-X_{1}^{-}, y_{2}, X_{1},-y_{1},-X_{1}^{-}, y_{3}, X_{1}, \cdots\right]=\lim \left[0, X_{n}\right], \\
X_{n+1}=\left(X_{n}, y_{n},-X_{n}^{-}\right) .
\end{array}
$$

We have shown such patterns for analogs of Hurwitz numbers as well as various algebraic quantities in our papers in the bibliography. Now we exploit these symmetries to compute the quadratic exponents for them.

\section{5. $p=2$ : 'REPEAT' SYMMETRY AND QUADRATIC EXPONENTS}

5.1. General set-up. If $p=2$ and $X_{1}=X_{1}^{-}$, then $X_{n}=-X_{n}^{-}$and the folding symmetry becomes a 'repeat' or 'doubling' symmetry, and thus they are approximated well by periodic continued fractions $\left[0, X_{n}, y_{n}, X_{n}, y_{n}, X_{n}, y_{n}, \cdots\right]$, which represent quadratic irrationalities. 
We use this simple observation to calculate $E_{2}(\alpha)$ for some transcendental and algebraic $\alpha$ 's of interest.

First consider for arbitrary $q$ and a tuple $X_{1}$ and sequence $y_{n}$ of partial quotients the continued fraction

$$
\alpha=\left[0, X_{1}, y_{1}, X_{1}, y_{2}, X_{2}, \cdots\right]=\lim \left[0, X_{n}\right], X_{n+1}=\left[X_{n}, y_{n}, X_{n}\right],
$$

and periodic, thus quadratic, approximations for it:

$$
\beta_{n}=\left[0, X_{n}, y_{n}, X_{n}, y_{n}, \cdots\right]=\left[0, X_{n}, y_{n}+\beta_{n}\right] .
$$

Let us write the $i$-th convergent of $\alpha$ as $p_{i} / q_{i}$ with the usual conventions, so that if $\left[0, X_{n}\right]$ has length $m+1$, then

$$
\left[0, X_{n}\right]=\frac{p_{m}}{q_{m}},\left[0, X_{n}, y_{n}\right]=\frac{p_{m+1}}{q_{m+1}},\left[0, X_{n}, y_{n}, X_{n}\right]=\frac{p_{2 m+1}}{q_{2 m+1}},
$$

so that $\operatorname{deg} q_{2 m+1}=2 \operatorname{deg} q_{m}+\operatorname{deg} y_{n}$.

Now $\left[0, X_{n}, y_{n}, X_{n}\right]$ is the portion common to the expansion of $\alpha$ and $\beta_{n}$. Hence, by (1), $\beta_{n}=\left(\left(y_{n}+\beta_{n}\right) p_{m}+p_{m-1}\right) /\left(\left(y_{n}+\beta_{n}\right) q_{m}+q_{m-1}\right)$, or equivalently,

$$
q_{m} \beta_{n}^{2}+\left(y_{n} q_{m}-p_{m}+q_{m-1}\right) \beta_{n}-\left(y_{n} p_{m}+p_{m-1}\right)=0,
$$

so that

$$
\log _{q} H\left(\beta_{n}\right)=\operatorname{deg} y_{n} q_{m}-\operatorname{deg} \operatorname{gcd}\left(q_{m}, y_{n} p_{m}+p_{m-1}, q_{m-1}-p_{m}\right) \leq \operatorname{deg} y_{n} q_{m} .
$$

The inequality becomes equality if $q_{m}, q_{m-1}+p_{m}, y_{n} p_{m}+p_{m-1}$ are relatively prime.

On the other hand, using two identities in (1), we see

$$
\begin{aligned}
\alpha-\beta_{n} & =\frac{\left(y_{n+1}+\alpha_{2 m+3}^{-1}\right) p_{2 m+1}+p_{2 m}}{\left[\left(y_{n+1}+\alpha_{2 m+3}^{-1}\right) q_{2 m+1}+q_{2 m}\right]}-\frac{\left(y_{n}+\beta_{n}\right) p_{2 m+1}+p_{2 m}}{\left[\left(y_{n}+\beta_{n}\right) q_{2 m+1}+q_{2 m}\right]} \\
& =\frac{\left(y_{n+1}+\alpha_{2 m+3}^{-1}\right)-\left(y_{n}+\beta_{n}\right)}{[\cdots][\cdots]} .
\end{aligned}
$$

Hence the non-Archimedean nature of the absolute values implies that if $\operatorname{deg} y_{n+1}>$ $\operatorname{deg} y_{n}$, then

Hence

$$
\left|\alpha-\beta_{n}\right|=\frac{1}{\left|y_{n} q_{2 m+1}^{2}\right|}
$$

$$
E_{2}(\alpha) \geq \lim \frac{\operatorname{deg} y_{n}+2 \operatorname{deg} q_{2 m+1}}{\operatorname{deg} y_{n}+\operatorname{deg} q_{m}}=3+\lim \frac{\operatorname{deg} q_{m}}{\operatorname{deg} y_{n}+\operatorname{deg} q_{m}} .
$$

Often in our examples, $E=E(\alpha)=2+\lim \operatorname{deg} y_{n} / \operatorname{deg} q_{m}$. Then the above bound implies $E_{2}(\alpha) \geq 3+1 /(E-1)$.

We summarize these calculations as

Theorem 2. Let $p=2$ and let $\alpha$ be as above with $\operatorname{deg} y_{n}$ increasing and $E:=$ $E(\alpha)=2+\lim \operatorname{deg} y_{n} / \operatorname{deg} q_{m}$. Then $E_{2}(\alpha) \geq 3+1 /(E-1)$.

5.2. Application to exponential. Let us apply this now, first to the transcendental exponential values.

Theorem 3. Let $p=2$. With the notation as in the previous theorem, we have $E_{2}(e(1 / f)) \geq 3+1 /(q-1)$.

Proof. Put $\alpha=e(1 / f)-1 / f$ and $y_{n}=-f^{q^{n}(q-2)} D_{n+1} / D_{n}^{2}$. Then the hypothesis of the theorem above is satisfied with $E=q$, by the formulas in the proof of Theorem 1. 
It is conceivable that the lower bound for $E_{2}$ in the last theorem can often (for example, say for $q=2$ ) be an equality, since for the quadratic expansion $\beta$ to give a good approximation to $\alpha$, several first partial quotients have to match and we have to do calculation as above, so the maximum matching at the fastest approximation spots that we have used probably leads to the exact value of $E_{2}$. But we have not been able to verify this rigorously. Let us first see that the approximating sequence $\beta_{n}$ indeed approximates to the exponent claimed, at least for $q=2$.

For $p=2$, by [T92, p. 153], we have (with $m=2^{n}-1$ ),

$$
\begin{gathered}
q_{m-1}=p_{m}, q_{m}=f^{q^{n}} D_{n}, y_{n}=f^{q^{n}(q-2)} \frac{D_{n+1}}{D_{n}^{2}}, \\
p_{m}=\sum_{1}^{n} f^{q^{n}-q^{i}} \frac{D_{n}}{D_{i}}, \quad p_{m-1}=\left(p_{m}+1\right)^{2} / q_{m} .
\end{gathered}
$$

Thus, we need to calculate the degree of the gcd of $q_{m}$ and $y_{n} p_{m}+p_{m-1}$.

Claim. The $\operatorname{gcd}$ is $G:=[1]^{2^{n}-1}$, if $q=2$ and $f=1$.

Proof. We now have $q_{m}=D_{n}, y_{n}=[n+1], p_{m}=\sum_{1}^{n} D_{n} / D_{i}$ and $p_{m-1}=$ $\sum_{1}^{n-1} D_{n} / D_{i}^{2}$. We first recall (see, e.g., [T04, Sec. 2.5]) that $[i]$ is the product of all monic primes of degree dividing $i$ and $D_{i}$ is the product of all monic polynomials of degree $i$. Let $w$ be the prime of degree $d$ which divides the gcd, so that $w$ divides $D_{n}$ and hence $d \leq n$. But then $w$ divides all terms in the sum above for $y_{n} p_{m}+p_{m-1}$, except possibly $[n+1]\left(D_{n} / D_{n}\right)+D_{n} / D_{n-1}^{2}=[1]^{2^{n}}$, so that $d=1$. By symmetry, gcd will be a power of [1]. Note that $G$ is the largest power of [1] dividing $D_{n}$. On the other hand, the same can be said by replacing $D_{n}$ with

$$
y_{n} p_{m}+p_{m-1}=D_{n}\left(\frac{[n+1]}{D_{1}}+\sum_{i=1}^{n-1}\left(\frac{[n+1]}{D_{i+1}}+\frac{1}{D_{i}^{2}}\right)\right),
$$

since the first term in the bracket is prime to [1] and

$$
\frac{[n+1] D_{i}^{2}+D_{i+1}}{D_{i+1} D_{i}^{2}}=\frac{[n+1]+[i+1]}{D_{i+1}}=\frac{[n-i]^{2^{i+1}}}{D_{i+1}}
$$

is divisible by [1].

Hence, in this case $\operatorname{deg} \log _{q} H\left(\beta_{n}\right)=2^{n+1}+n 2^{n}-\left(2^{n}-1\right) * 2=n 2^{n}+2$, and we see that the approximation exponent for the approximating sequence $\beta_{n}$ is indeed equal to (rather than just $\geq$ ) the claimed value. It is easy to see that the same works for $q=2$ and $e(1 / f)-1 / f$. In general, for any $q=2^{n}$ and any nonzero $f$, the author expects (but has not checked the details) that the gcd similarly has a degree of lower order of magnitude than the main term $n q^{n}$, and hence the lower bound is equality at least for the approximating sequence $\beta_{n}$.

As Voloch pointed out to the author, a straight generalization of Voloch's V88, Prop. 5] implies that if the ratio of the degrees of consecutive elements of the approximating sequence $r_{i}$ of degree $d$ elements approximating $\alpha$ tends to $R$ and they approximate to the exponent $E$, with $E>d(\sqrt{R}+1)$, then $E$ is in fact the exponent $E_{d}(\alpha)$.

The only change in the proof there given for $d=1$ is the replacement of $H\left(r_{1}+r_{2}\right) \leq H\left(r_{1}\right)+H\left(r_{2}\right)$ there by $\leq d\left(H\left(r_{1}\right)+H\left(r_{2}\right)\right)$, where $r_{i}$ is the approximation of degree $d$. 
Unfortunately, $\operatorname{deg} \log _{q} H\left(\beta_{n+1}\right) / \operatorname{deg} \log _{q} H\left(\beta_{n}\right)$ tends to $q$ as $n$ tends to infinity and $3+1 /(q-1)<2(\sqrt{q}+1)$, so we cannot get the exact value of the exponent by a straight application of this result.

5.3. Application to algebraic quantities. Next, we apply it to algebraic $\alpha$ 's, as in (5), (6), where we can get even better lower bounds by constructing examples with many repetitions as follows.

Let $p=2$. Choose some palindromic vector $X_{1}=X_{1}^{-}$of partial quotients and $y_{i}$ polynomials of degree at least 1 . Suppose $\left[0, X_{1}\right]=a / f$. Then by the folding lemma, we have

$$
\alpha=\frac{a}{f}+\frac{1}{y_{1} f^{2}}+\frac{1}{y_{2} y_{1}^{2} f^{4}}+\frac{1}{y_{3} y_{2}^{2} y_{1}^{4} f^{8}}+\cdots,
$$

as long as the hypothesis of the folding lemma holds at each stage, namely denominators claimed do not get reduced. This can often be arranged easily. For example, choose $X=f$ to be a singleton, so that $a=1$. Choose all $y_{i}$ 's to be powers of the same irreducible and $f$ to be a power of the (same or another) irreducible.

We give some such family of examples and leave variations to the reader.

We fix integers $m, r>1$ and let $y$ be an irreducible and $y_{j}:=y$ if $m$ does not divide $j$ and $y_{j}:=y^{r}$ if $m$ divides $j$. Let $B_{i}:=y_{m+i} y_{m+i-1}^{2} \cdots y_{i+1}^{2^{m-1}}$.

By looking at the positions at which $y^{r}$ occurs, we see that the continued fraction is not eventually periodic and thus $\alpha$ is not quadratic. We decompose $\alpha$ as above, with $a=1$, as $\sum \alpha_{i}$, where $i$ runs through 0 and $m-1$ and $\alpha_{i}$ consists of the subsum containing terms of the above series with index (starting with 0) $i$ modulo $m$. We thus see that $\alpha_{i}^{2^{m}} / B_{i}-\alpha_{i}=1 /\left(y_{i} \cdots y_{1}^{2^{i-1}} f^{2^{i}}\right)$. Thus $\operatorname{deg} \alpha \leq 2^{m^{2}}$.

We have a bounded partial quotient sequence, so that $E_{1}=2$. On the other hand, if we again approximate by $\beta_{n}$, but now for $n$ 's which are congruent to 1 modulo $m$, we see that there is a $2^{m-1}$-fold repetition match of $X_{n}$, so that the same calculation as above now shows that $E_{2} \geq 2^{m}$. Note now that we need not have $\operatorname{deg} y_{n+1}>\operatorname{deg} y_{n}$, but we leave it to the reader to verify that it does not matter for the calculation.

We summarize these calculations as

Theorem 4. When $p=2$, given any $m>1$, there are explicit families (described above) of algebraic irrationals $\alpha$ of degree greater than 2 and less than $2^{m^{2}}$, with a bounded partial quotient sequence, so that $E_{1}(\alpha)=2$, and with $E_{2}(\alpha) \geq 2^{m}$ (thus breaking the analog of the Schmidt bound of 3).

Remarks. (1) The calculation above implies that the doubling symmetry cannot happen for any $\alpha$ of degree 3; otherwise the Liouville bound of 3 for the quadratic exponent would be exceeded.

(2) It is quite possible that the Liouville bound for the quadratic exponent can be reached for a properly constructed variant of the family above.

(3) We deal with rational and quadratic approximations above. The next cubic case falls under the more general so-called class I elements, namely those $\alpha$ whose $q^{k}$-th power is an integral Mobius transformation of $\alpha$. This is exactly the case, where a lot is known about continued fractions and there are automata/transducer tools generating them (starting from BS76]; see references in [S00, T04]). So it is conceivable that progress can be made in this way. 


\section{6. $p>2$ : 'HALF-REPEAT' SYMMETRY AND QUADRATIC EXPONENTS}

Again, as before, let $\alpha=\left[0, X_{1}, y_{1},-X_{1}^{-}, y_{2}, X_{1},-y_{1},-X_{1}^{-}, y_{3}, X_{1}, \cdots\right]$ and

$$
\beta_{n}=\left[0, X_{n}, y_{n}, X_{n}, y_{n}, \cdots\right]=\left[0, X_{n}, y_{n}+\beta_{n}\right] \text {. }
$$

This time there is a half-repeat: after $X_{n}, y_{n}$ only the $X_{n-1}$ part is repeated in $\alpha$ and thus only that part matches with $\beta_{n}$ entries.

As before, let us write the $i$-th convergent of $\alpha$ as $p_{i} / q_{i}$ with the usual conventions, so that if $\left[0, X_{n}\right]$ has length $m+1$, then

$$
\left[0, X_{n-1}, y_{n-1},-X_{n-1}^{-}\right]=\left[0, X_{n}\right]=\frac{p_{m}}{q_{m}},\left[0, X_{n}, y_{n}\right]=\frac{p_{m+1}}{q_{m+1}}
$$

and

$$
\left[0, X_{n-1}, y_{n-1},-X_{n-1}^{-}, y_{n}, X_{n-1}\right]=\frac{p_{*}}{q_{*}},
$$

where $*=m+1+(m-1) / 2$. As before, $\log _{q} H\left(\beta_{n}\right) \leq \operatorname{deg} y_{n} q_{m}$.

By calculation as before, using the two identities in (1), we see

$$
\alpha-\beta_{n}=\frac{\left(y_{n-1}+\cdots\right)-\left(-y_{n-1}+\cdots\right)}{\left(\left(y_{n-1}+\cdots\right) q_{*}+q_{*-1}\right)\left(\left(-y_{n-1}+\cdots\right) q_{*}+q_{*-1}\right)},
$$

and thus

$$
\left|\alpha-\beta_{n}\right|=\frac{1}{\left|y_{n-1} q_{*}^{2}\right|}
$$

Hence, as before, we get

$$
E_{2}(\alpha) \geq \lim \frac{\operatorname{deg} y_{n-1}+2 \operatorname{deg} q_{*}}{\operatorname{deg} y_{n}+\operatorname{deg} q_{m}} .
$$

Let us write $E$ for $E(\alpha)$ and $Q$ for $\operatorname{deg} q_{(m-1) / 2}$, so that in our examples (those in [T04, p. 316] or $e(1 / f)$ above), $\lim \operatorname{deg} y_{n} / \operatorname{deg} q_{m}=\lim \operatorname{deg} y_{n-1} / Q=E-2$. Also, $\operatorname{deg} q_{m}=2 Q+\operatorname{deg} y_{n-1}$ and $\operatorname{deg} q_{*}=3 Q+\operatorname{deg} y_{n}+\operatorname{deg} y_{n-1}$. Putting these in the $E_{2}$ bound above, we get

$$
\begin{aligned}
E_{2}(\alpha) & \geq 2+\frac{Q[(E-2)+2(3+(2+(E-2))(E-2)+(E-2))]}{Q[(2+(E-2))(E-2)+(2+(E-2))]} \\
& =2+\frac{1}{E-1} .
\end{aligned}
$$

Theorem 5. If $p>2$ and $\alpha$ is as above, with $E=E(\alpha)=2+\lim \operatorname{deg} y_{n} / \operatorname{deg} q_{m}$, then $E_{2}(\alpha) \geq 2+1 /(E-1)$.

As before, applied to the exponential case, this implies,

Theorem 6. If $p>2$, then $E_{2}(e(1 / f)) \geq 2+1 /(q-1)$.

Remarks. For general $p$ and $q=p^{k}$, by choosing increasing $y_{i}$, we can construct, by the same method as in the last section, several algebraic explicit continued fractions, say by groupings, for large enough $n, y_{n+i} y_{n+i-1}^{2} \cdots y_{n+i-k+1}^{2^{k-1}} f^{2^{n+i}}=A_{i} f^{p^{n+i}}$, with $A_{i}$ depending on $i \bmod k$. We can also have longer initial segment variation. These are just variations on examples in T96. These examples will not have bounded partial quotients now, but the negative reversal symmetry and Theorem 5 above apply. 


\section{ExPONENT OF ANALOG OF $\pi$}

Consider $\pi:=\prod_{n=1}^{\infty}\left(1-t^{1-q^{n}}\right)^{-1} \in K_{\infty}$. It can be considered (up to rational multiple) as an analog of the usual real number $\pi$; see [T04, p. 47].

Theorem 7. For $\pi$ as above, $E(\pi) \geq q-1$, with equality when $q \geq 5$.

Proof. If we just use truncation approximations, for $1 / \pi$ from the product formula given above, we see they have denominator of degree $(q-1)+\cdots+\left(q^{n}-1\right)$ asymptotic to $q^{n+1} /(q-1)$ so that the exponent is at least $q-1$. If we use the proposition of Voloch [V88, Prop. 5] (see T04, Lemma 9.3.3] or quote above), we get the equality, for $q \geq 5$, as $q-1>\sqrt{q}+1$ then.

Remarks. (1) We can get similar calculations for some zeta values and other quantities of interest by the same method.

(2) What are the exponents and the higher exponents known/conjectured for the usual $e, \pi$ ? It is known that $E(e)=2$, as follows e.g. by Euler's continued fraction. We know Sa08 that $E(\pi)<7.61$. It seems that the best known lower bound is the trivial bound 2 and that the exact higher exponents are not known for $e$ or $\pi$. The author does not know of references for the best known lower/upper bounds.

\section{EXPONENT VARIATION WITHIN A FIELD}

We close with a few simple remarks on the question of exponent variation within a function field.

(I) We know of (e.g. BS76 ) a degree 3 algebraic element $\alpha$ with bounded partial quotients, so that its exponent is 2 . We claim that any irrational in the function field generated by $\alpha$ over $K$ has bounded partial quotients (and thus exponent 2): We know that any such element is of the form $\beta:=k_{2} \alpha^{2}+k_{1} \alpha+k_{0}$, with $k_{i} \in K$, but then using the cubic equation satisfied by $\alpha$, it is straightforward manipulation to see that $\beta$ can also be written as a Mobius transform of $\alpha$ with coefficients in $K$ (and nonzero determinant), and thus the claim follows from BS76.

(II) We also know of (e.g. BS76]) a degree 3 element with exponent greater than 2, and thus it generates an extension of $K$ all of whose irrational elements have (the same) exponent greater than two by the argument above.

(III) Taking the compositum of fields containing different exponent elements, it is of course much easier to get mixed exponent fields.

\section{REFERENCES}

[BS76] L. Baum and M. Sweet, Continued fractions of algebraic power series in characteristic 2, Ann. of Math 103 (1976), 593-610. MR0409372 (53:13127)

[BG06] E. Bombieri and W. Gubler, Heights in Diophantine Geometry, Cambridge U. Press, Cambridge (2006). MR2216774 (2007a:11092)

[B04] Y. Bugeaud, Approximation by algebraic numbers, Cambridge U. Press (2004). MR2136100 (2006d:11085)

[KTV00] M. Kim, D. Thakur and J. F. Voloch, Diophantine approximation and deformation, Bull. Soc. Math. France 128 (2000), 585-598. MR1815399 (2001k:11134)

[M49] K. Mahler, On a theorem of Liouville in fields of positive characteristic, Can. J. Math. 1 (1949), 397-400. MR0031497 (11:159e)

[dM70] B. de Mathan, Approximations diophatiennes dans un corps local, Bull. Soc. Math. France Suppl. Mem. 21:93 (1970). MR0274396 (43:161)

[MF73] M. Mendes France, Sur les continues fractions limitées, Acta Arith. 23 (1973), 207-215. MR0323727 (48:2083) 
[R03] D. Roy, Approximation to real numbers by cubic algebraic integers, Annals of Math. 158 (2003), 1081-1087. MR2031862 (2004k:11110)

[Sa08] V. Kh. Salikhov, On the irrationality measure of $\pi$, Communications of Moscow Math. Soc. 63 (2008) 570-572. MR2483171 (2010b:11082)

[S80] W. Schmidt, Diophantine approximation, Lecture Notes in Math. 785, Springer Verlag, Berlin (1980). MR568710 (81j:10038)

[S00] W. Schmidt, On continued fractions and diophantine approximation in power series fields, Acta Arith. XCV.2 (2000), 139-166. MR.1785412 (2001j:11063)

[T92] D. Thakur, Continued fraction for the exponential for $F_{q}[t]$, J. Number Theory 41 (1992), 150-155. MR1164793 (93d:11131)

[T96] D. Thakur, Exponential and continued fractions, J. Number Theory 59 (1996), 248-261. MR 1402608 (97h:11145)

[T97] D. Thakur, Patterns of continued fractions for the analogues of e and related numbers in the function field case, J. Number Theory 66 (1997), 129-147. MR1467193 (98d:11145)

[T99] D. Thakur, Diophantine approximation exponents and continued fractions for algebraic power series, J. Number Theory 79 (1999), 284-291. MR 1728151(2000j:11104)

[T04] D. Thakur, Function Field Arithmetic, World Scientific, NJ (2004). MR2091265 (2005h:11115)

[T09] D. Thakur, Approximation exponents in function fields, Analytic Number Theory, Essays in honor of Klaus Roth, ed. by W. Chen, T. Gowers, H. Halberstam, W. Schmidt, R. Vaughn, Cambridge U. Press, Cambridge (2009), 421-435. MR 2508661 (2010b:11079)

[V88] J. F. Voloch, Diophantine approximation in finite characteristic, Period. Math. Hungar. 19 (1988), 217-225. MR 961018 (89h:11045)

[W] M. Waldschmidt, Report on some recent advances in Diophantine approximation, to be published (Springer) in special volume in honor of Serge Lang. Also at people.math.jussieu.fr/ ${ }^{\sim}$ miw/articles/pdf/miwLangeMemorialVolume.pdf

Department of Mathematics, University of Arizona, 617 N. Santa Rita Avenue, TucSON, ARIZONA 85721

E-mail address: thakur@@math.arizona.edu 